
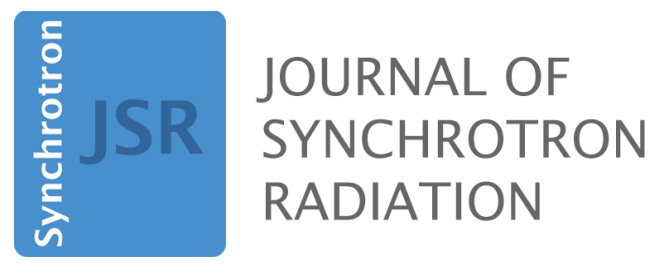

Volume 25 (2018)

Supporting information for article:

More are better, but the details matter: combinations of multiple Fresnel zone plates for improved resolution and efficiency in X-ray microscopy

Kenan Li and Chris Jacobsen 


\title{
Supplementary material to "More are better, but the details matter: combinations of multiple Fresnel zone plates for improved resolution and efficiency in x-ray microscopy"
}

\author{
KENAN Li ${ }^{a, b 1}$ AND ChRIS JACOBSEN ${ }^{b, c, d *}$ \\ ${ }^{a}$ Applied Physics, Northwestern University, Evanston, IL 60208, USA, ${ }^{b}$ Advanced \\ Photon Source, Argonne National Laboratory, Argonne, IL 60439, USA, \\ ${ }^{c}$ Department of Physics $\&$ Astronomy, Northwestern University, Evanston, IL \\ 60208, USA, and ${ }^{d}$ Chemistry of Life Processes Institute, Northwestern University, \\ Evanston, IL 60208, USA. E-mail: cjacobsen@anl.gov
}

\section{Fresnel zone plates; X-ray microscopy; X-ray optics}

In this Supplement, we follow prior work on understanding the effects of alignment errors on stacked zone plates (Vila-Comamala et al., 2013; Gleber et al., 2014) by providing a more detailed look at the effects on the focal spot. This is done by considering the first or upstream zone plate to have a diameter $d=45 \mu \mathrm{m}$, and an outermost zone width of $d r_{N}=25 \mathrm{~nm}$, thus giving a focal length of $f=9074 \mu \mathrm{m}$ at $10 \mathrm{keV}$ and a depth of focus of $2 \delta_{z}=25 \mu \mathrm{m}$. Each individual zone plate is assumed to have a thickness of $t=500 \mathrm{~nm}$, giving a theoretical first order focusing efficiency of $5.0 \%$ at $10 \mathrm{keV}$ for a single zone plate and $32.7 \%$ for $n_{\mathrm{zp}}=4$ such zone plates placed in close proximity.

In order to illustrate the effects of larger separation distances between zone plates, Fig. 1 shows $n_{\mathrm{zp}}=4$ of the above zone plates stacked at intervals of $\Delta z=10 \mu \mathrm{m}$

${ }^{1}$ Present address: SLAC, Menlo Park, CA 94025, USA 
(A) so that the net separation between upstream and downstream zone plates is 30 $\mu \mathrm{m}$, or at intervals of $\Delta z=100 \mu \mathrm{m}$ leading to a net separation of $300 \mu \mathrm{m}(\mathrm{B})$. The intervals with case (A) are less than the depth of focus of $25 \mu \mathrm{m}$, so one obtains a sharp focus with only a $24.8 \%$ increase in the FWHM probe width, but with a reduction in efficiency to $15.6 \%$ versus the close-proximity case of $32.7 \%$. When the intervals are increased well beyond the depth of focus (B), four distinct axial foci are produced so it is difficult to talk about a common FWHM probe size or a simple focusing efficiency value. However, if one allows the $i^{\text {th }}$ zone plate to have its diameter $d_{i}$ adjusted so as to focus to the same point for all four zone plates, one obtains a fine focus with $\delta_{\mathrm{FWHM}}=25.4 \mathrm{~nm}$ and a net efficiency of $39.3 \%$.
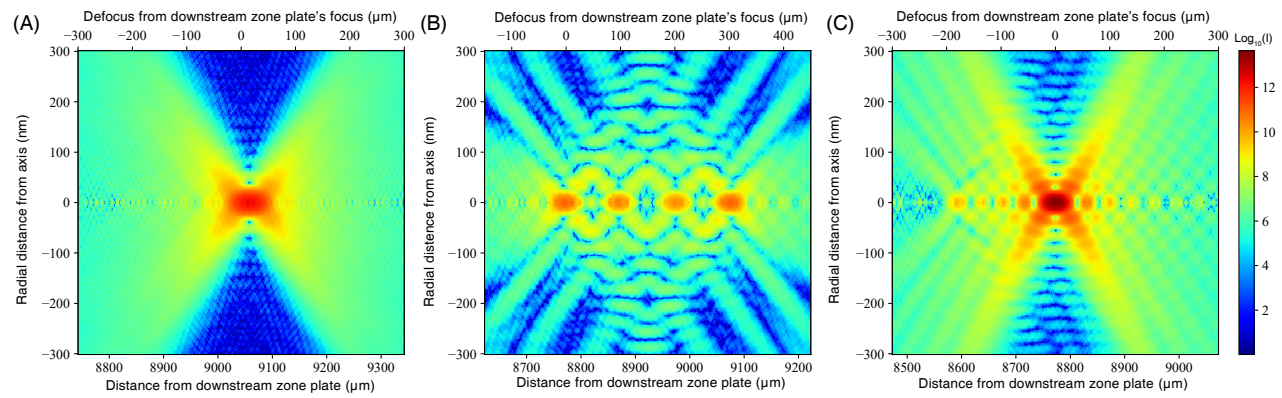

Fig. 1. Simulation of beyond-near-field stacking $n_{\mathrm{zp}}=4$ gold zone plates. The upstream zone plate is assumed have a diameter of $d=45 \mu \mathrm{m}$ and an outermost zone width of $d r_{N}=25 \mathrm{~nm}$, giving a focal length of $9074 \mu \mathrm{m}$ at an x-ray energy of $10 \mathrm{keV}$ and a depth of focus of $2 \delta_{z}=40.3 \mu \mathrm{m}$. If we stack four zone plates (each of $t=500 \mathrm{~nm}$ thick gold) in close proximity, the theoretical first-diffraction-order focusing efficiency is $32.7 \%$. If we instead stack these zone plates with $\Delta z=10 \mu \mathrm{m}$ separation (A), the diffraction efficiency is reduced to $15.6 \%$ and the full-width at half-maximum focal spot size is slightly enlarged from $\delta_{\mathrm{FWHM}}=26 \mathrm{~nm}$ to $32.2 \mathrm{~nm}$. (B) If the separation is increased to $100 \mu \mathrm{m}$ which is larger than the depth of focus, four distinct focal points are produced along the beam direction, and the separation between foci is the same as the zone plate separation. (C) If the zone plates are separated by $\Delta z=100 \mu \mathrm{m}$ but the diameter $d_{i}$ of the $i^{\text {th }}$ zone plate is adjusted so that it focuses to the position of the first, upstream zone plate, one obtains a higher efficiency of $39.3 \%$ and a tighter focal spot with $\delta_{\mathrm{FWHM}}=25.4 \mathrm{~nm}$.

Several studies (Simpson \& Michette, 1983; Pratsch et al., 2014) have shown that the focusing properties of single Fresnel zone plates begin to degrade when zones IUCr macros version 2.1.10: 2016/01/28 
are mispositioned by about a third to a half of their width (half a zone width or $\sim d r_{N} / 2$ for the outermost zone corresponds to the Rayleigh quarter-wave criterion). One would expect something similar with lateral alignment in zone plate stacking (Shastri et al., 2001; Maser et al., 2002; Snigireva et al., 2007; Kagoshima et al., 2011; Gleber et al., 2014), so that the lateral error $\Delta x_{i}$ should be small compared to the outermost zone width $d r_{N}$. An additional requirement in zone plate stacking is that the longitudinal error $\Delta z_{i}$ should be small compared to the depth of focus DoF, which is most restrictive for the most downstream zone plate due to its finer zone width. These alignment errors are shown in Fig. 2. The role of both errors in zone plate stacking has been examined previously in both simulation and experiment (Gleber et al., 2014).

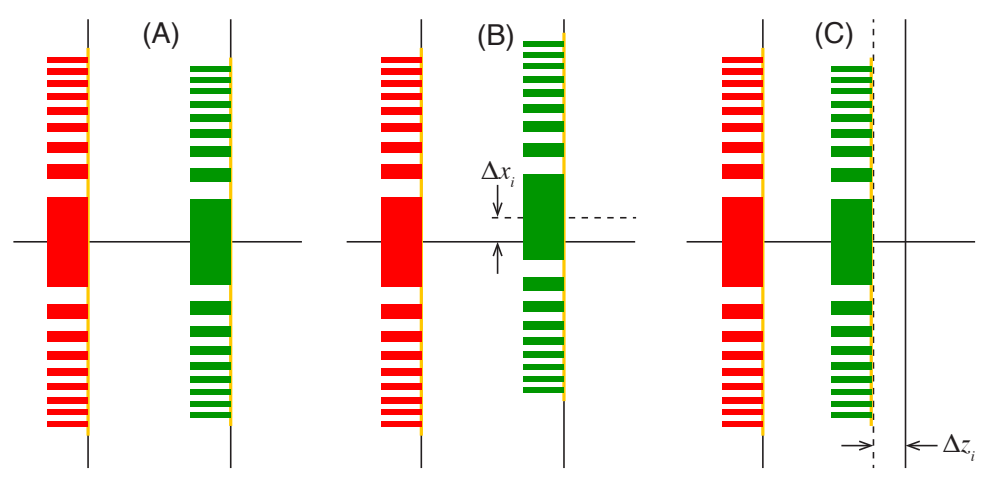

Fig. 2. Zone plate stacking with no error (A), a transverse misalignment $\Delta x_{i}(\mathrm{~B})$, and a longitudinal misalignment $\Delta z_{i}(\mathrm{C})$.

For zone plate transverse misalignment, Fig. 3 shows simulations of the stacking of two zone plates with $50 \mu \mathrm{m}$ separation and misalignment $\Delta x_{i}$ ranging from 0 to $2 d r_{N, 1}$. We see that a misalignment of $\Delta x_{i}=1.0 d r_{N, 1}$ leads to an elliptical focal spot, while a misalignment of $\Delta x_{i}=2.0 d r_{N, 1}$ leads to split focal spots within the focal plane (the transition to multiple focal spots also leads to fringes in the far-field diffraction pattern (Shastri et al., 2001; Maser et al., 2002; Gleber et al., 2014)). Similar to the IUCr macros version 2.1.10: 2016/01/28 
case for zone placement errors in single zone plates, when the transverse misalignment exceeds about $\Delta x_{i} \sim d r_{N, 1} / 3$, Fig. 3(D) shows that the peak intensity of the focus begins to decrease and the FHWM spot size begins to increase.
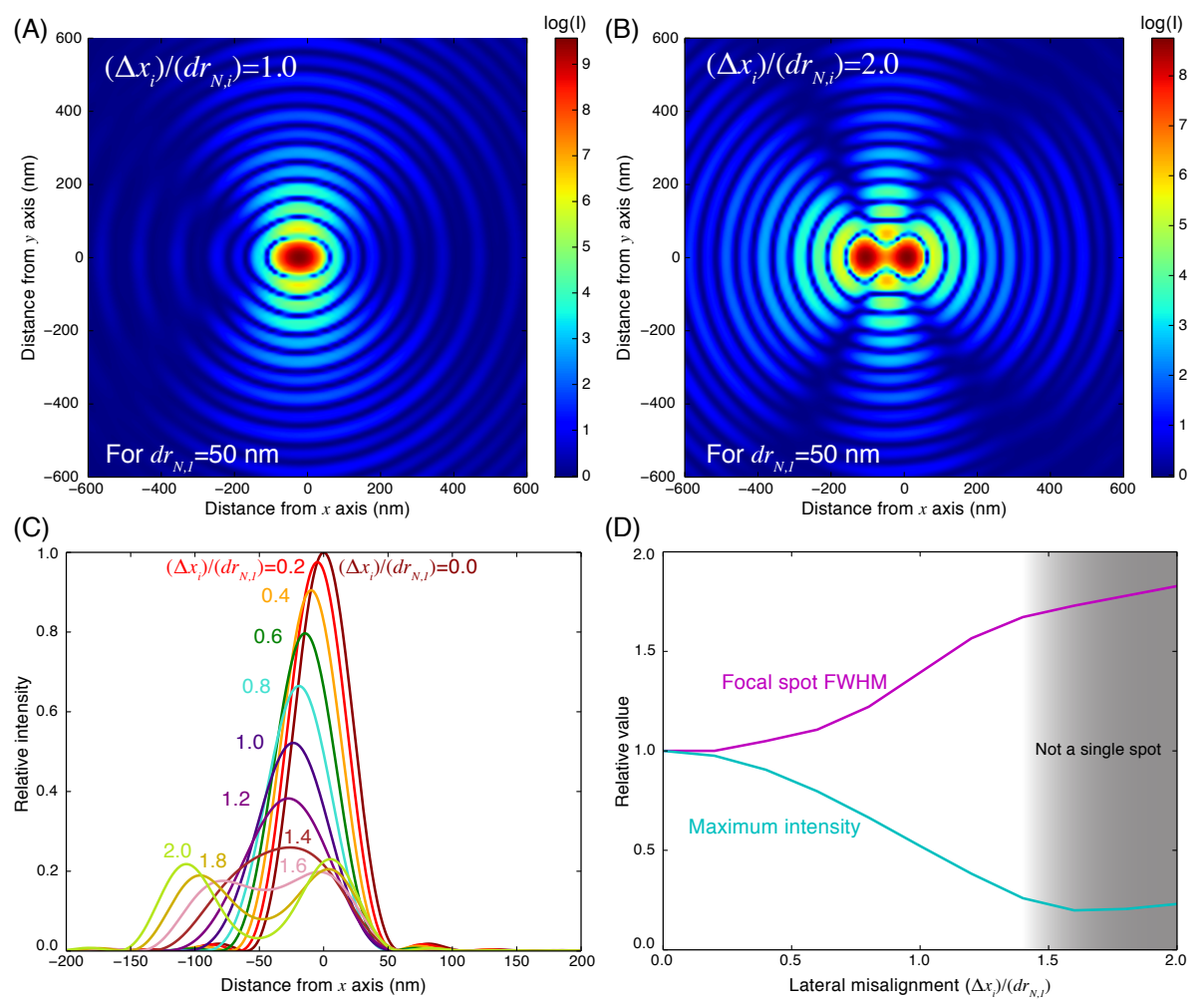

Fig. 3. Transverse misalignment in zone plate stacking. At top is shown the focal spot intensity with a transverse misalignment between two zone plates of $\Delta x_{i}=1.0 d r_{N, 1}$ (A) and $\Delta x_{i}=2.0 d r_{N, 1}$ (B). At bottom left (C) is shown a series of plots of the transverse intensity profile for a range of transverse misalignments $\Delta x_{i}$ ranging from $0.0 d r_{N, 1}$ to $2.0 d r_{N, 1}$, while at right (D) we show the relative change in both the fullwidth at half maximum (FWHM) focal spot size, and the maximum intensity. As can be seen, transverse alignment errors $\Delta x_{i}$ of about half of the outermost zone width, or $\sim 0.5 d r_{N, 1}$, lead to degradations in zone plate focusing. These simulations used two zone plates, each with diameter $d=45 \mu \mathrm{m}$ and thickness $t_{i}=1 \mu \mathrm{m}$, and an outermost zone width on the first zone plate of $d r_{N, 1}=50 \mathrm{~nm}$. The separation between the two zone plates was $\Delta z=50 \mu \mathrm{m}$, and a photon energy of $10 \mathrm{keV}$ was used.

For zone plate longitudinal misplacement, Fig. 4 shows simulations of alignment errors $\Delta z_{i}$ ranging from 0 to 1 DoF. We see that a misplacement of $\Delta z_{i}=1.0 \mathrm{DoF}$ IUCr macros version 2.1.10: 2016/01/28 
leads to an extended focal spot in depth, while a misplacement of $\Delta z_{i}=2.0 \mathrm{DoF}$ leads to a split focal spot along the longitudinal axis as well as a wider but hollow focal spot at the midpoint. The derivative of a zone plate's focal length $f$ of with respect to diameter $d$ gives

$$
\Delta f=(\Delta d) \frac{d r_{N}}{\lambda}
$$

while the depth of focus can be arranged to give $\left(d r_{N} / \lambda\right)=\operatorname{DoF} /\left(4.88 d r_{N}\right)$, which when substituted into Eq. 1 gives

$$
\frac{\Delta f}{\operatorname{DoF}}=\frac{\Delta d}{4.88 d r_{N}}
$$

If the diameter $d$ is pulled back by half of the outermost zone width $d r_{N}$ (a Rayleigh quarter-wave deviation) on each side of the optical axis, the net change in diameter is $\Delta d=d r_{N}$ so $(\Delta f)=\operatorname{DoF} / 4.88 \simeq 0.21 \mathrm{DoF}$ corresponds to the Rayleigh quarter wave criterion for longitudinal misalignment of the two zone plates. As can be seen in Figs. 4(C) and (D), this gives a slightly over-restrictive tolerance on longitudinal alignment.

IUCr macros version 2.1.10: 2016/01/28 

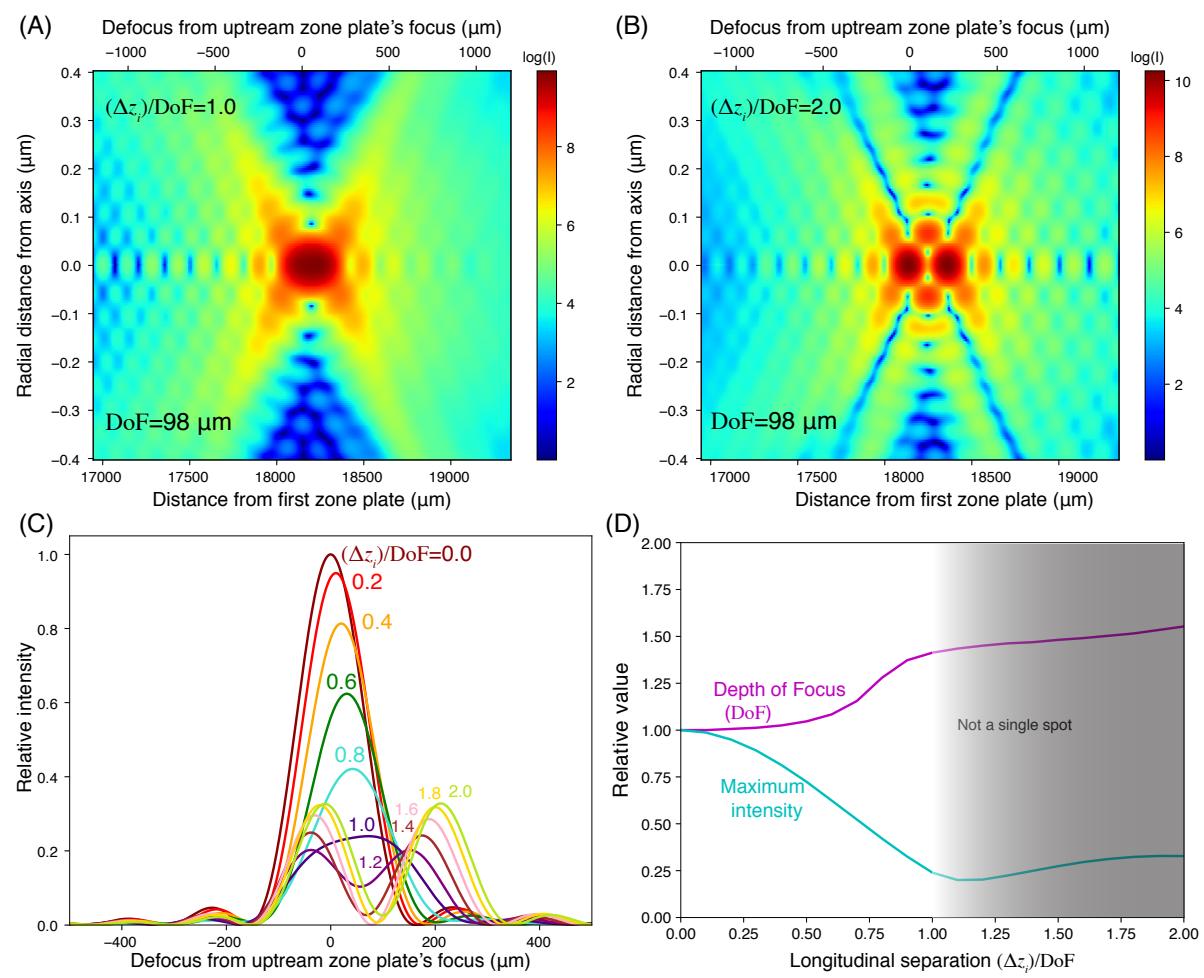

Fig. 4. Longitudinal misalignment in zone plate stacking as a function of depth of focus, which is $\mathrm{DoF}=98.4 \mu \mathrm{m}$ in this case. At top is shown the longitudinal-radial intensity distribution for a misalignment of $\Delta z_{i}=1.0 \mathrm{DoF}(\mathrm{A})$ and for $\Delta z_{i}=2.0 \mathrm{DoF}$ (B); in the latter case, two distinct focal spots appear along the longitudinal axis. At bottom left $(\mathrm{C})$ is shown a series of plots of the focus intensity profile for a range of longitudinal misalignments $\Delta z_{i}$ ranging from $0.0 \mathrm{DoF}$ to $2.0 \mathrm{DoF}$, while at right (D) we show the relative change in both the full-width at half maximum (FWHM) focal spot size, and the maximum intensity. As can be seen, longitudinal alignment errors $\Delta z_{i}$ of about a third of a depth of focus, or $\sim 0.3 \mathrm{DoF}$, lead to degradations in zone plate focusing. These simulations used two zone plates, each with diameter $d=45 \mu \mathrm{m}$ and thickness $t_{i}=1 \mu \mathrm{m}$, and an outermost zone width on the first zone plate of $d r_{N, 1}=50 \mathrm{~nm}$. The separation between the two zone plates was $\Delta z=50$ $\mu \mathrm{m}$, and a photon energy of $10 \mathrm{keV}$ was used.

With a single thin zone plate or grating with 1:1 line:space ratio, only odd order diffractions and foci are produced. Even-order diffraction (such as second order) comes either from departures from a 1:1 line:space ratio, or volume diffraction effects (dynamic diffraction within gratings); both of these effects are shown in Fig. 5(A). Even-diffraction-order focusing is also increased when using stacked zone plates, as IUCr macros version 2.1.10: 2016/01/28 
shown in Fig. 5(B). This might be due to the additional high-spatial-frequency modulation imposed on the wavefield by the second zone plate.

IUCr macros version 2.1.10: 2016/01/28 
(A)
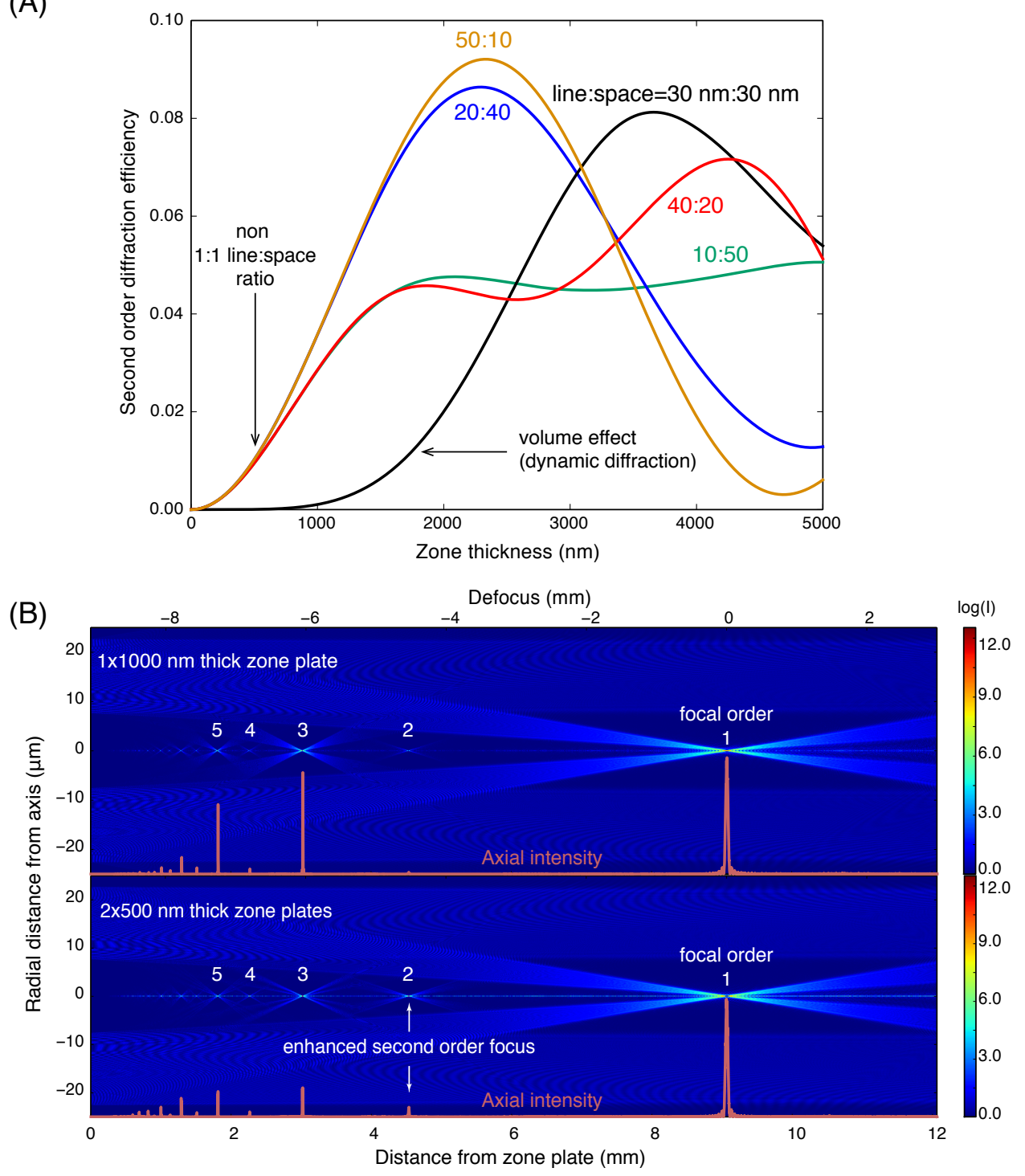

Fig. 5. Thin zone plates with a 1:1 line:space ratio show no second order diffraction. However, light in a second order focus can appear (A) when the line:space ratio is changed from 1:1, but also when volume diffraction effects begin to play a role (as calculated using the multislice method ( $\mathrm{Li}$ et al., 2017)). Light in the second order focus can also appear when one uses stacked zone plates. Shown here (B) is a comparison between a single $t=1000 \mathrm{~nm}$ thick zone plate, and two $500 \mathrm{~nm}$ thick zone plates separated by $50 \mu \mathrm{m}$. As before, these simulations are for $d=45$ $\mu \mathrm{m}$ diameter zone plates with $d r_{N}=25 \mathrm{~nm}$ outermost zone width and (for B) a line:space ratio of 1:1. An x-ray energy of $10 \mathrm{keV}$ was used.

\section{References}


Gleber, S. C., Wojcik, M., Liu, J., Roehrig, C., Cummings, M., Vila-Comamala, J., Li, K., Lai, B., Shu, D. \& Vogt, S. (2014). Optics Express, 22(23), 28142-28153.

Kagoshima, Y., Takano, H., Koyama, T., Tsusaka, Y. \& Saikubo, A. (2011). Japanese Journal of Applied Physics, 50(2).

Li, K., Wojcik, M. \& Jacobsen, C. (2017). Optics Express, 25(3), 1831-1846.

Maser, J., Lai, B. P., Yun, W., Shastri, S. D., Cai, Z., Rodrigues, W., Xu, S. \& Trackhtenberg, E. (2002). Proceedings SPIE, 4793, 74-81.

Pratsch, C., Rehbein, S., Werner, S. \& Schneider, G. (2014). Optics Express, 22(25), 3048230491.

Shastri, S. D., Maser, J. M., Lai, B. \& Tys, J. (2001). Optics Communications, 197(1-3), 9-14.

Simpson, M. J. \& Michette, A. G. (1983). Optica Acta, 30(10), 1455-1462.

Snigireva, I., Snigirev, A., Kohn, V., Yunkin, V., Grigoriev, M., Kuznetsov, S., Vaughan, G. \& Di Michiel, M. (2007). Physica Status Solidi A, 204(8), 2817-2823.

Vila-Comamala, J., Wojcik, M., Diaz, A., Guizar-Sicairos, M., Kewish, C. M., Wang, S. \& David, C. (2013). Journal of Synchrotron Radiation, 20(3), 397-404. 